\title{
Correction to: Influence of CYP2C19 genotypes for the effect of 1-month dual antiplatelet therapy followed by clopidogrel monotherapy relative to 12-month dual antiplatelet therapy on clinical outcomes after percutaneous coronary intervention: a genetic substudy from the STOPDAPT-2
}

\author{
Hirotoshi Watanabe ${ }^{1} \cdot$ Takeshi Morimoto $^{2} \cdot$ Manabu Ogita $^{3} \cdot$ Satoru Suwa $^{3} \cdot$ Masahiro Natsuaki $^{4}$. \\ Nobuhiro Suematsu ${ }^{5}$. Yorihiko Koeda ${ }^{6}$. Yoshihiro Morino ${ }^{6}$. Akira Nikaido ${ }^{7}$. Yoshiki Hata ${ }^{7}$. Masayuki Doi ${ }^{8}$. \\ Kiyoshi Hibi ${ }^{9}$ Kazuo Kimura ${ }^{9}$. Shunsuke Yoda ${ }^{10}$. Takeo Kaneko ${ }^{10}$. Koji Nishida ${ }^{11}$. Kazuya Kawai ${ }^{11}$. \\ Koji Yamaguchi $^{12}$. Tetsuzo Wakatsuki ${ }^{12}$. Norimasa Tonoike ${ }^{13} \cdot$ Masashi Yamamoto $^{13}$. Shogo Shimizu ${ }^{14}$. \\ Takao Shimohama ${ }^{15}$. Junya Ako ${ }^{15}$. Takeshi Kimura ${ }^{1}$ on behalf of STOPDAPT-2 Investigators
}

Published online: 12 February 2021

(c) Japanese Association of Cardiovascular Intervention and Therapeutics 2021

\section{Correction to: \\ Cardiovascular Intervention and Therapeutics https://doi.org/10.1007/s12928-020-00719-6}

In the original publication of the article, Fig. 4(B) and 4(C) were published incorrectly. The correct figures are given in this correction.

The original article can be found online at https://doi.org/10.1007/ s12928-020-00719-6.

Takeshi Kimura

taketaka@kuhp.kyoto-u.ac.jp

1 Department of Cardiovascular Medicine, Kyoto University Graduate School of Medicine, 54 Shogoin Kawahara-cho, Sakyo-ku, Kyoto 606-8507, Japan

2 Department of Clinical Epidemiology, Hyogo College of Medicine, Nishinomiya, Japan

3 Department of Cardiovascular Medicine, Juntendo University Shizuoka Hospital, Izunokuni, Japan

4 Department of Cardiovascular Medicine, Saga University, Saga, Japan

5 Department of Cardiovascular Medicine, Saiseikai Fukuoka General Hospital, Fukuoka, Japan

6 Division of Cardiology, Department of Internal Medicine, Memorial Heart Center, Iwate Medical University, Morioka, Japan

7 Department of Cardiology, Minamino Cardiovascular Hospital, Hachioji, Japan
8 Department of Cardiology, Kagawa Prefectural Central Hospital, Takamatsu, Japan

9 Division of Cardiology, Yokohama City University Medical Center, Yokohama, Japan

10 Department of Internal Medicine, Division of Cardiology, Shimonoseki City Hospital, Shimonoseki, Japan

11 Department of Cardiology, Chikamori Hospital, Kochi, Japan

12 Department of Cardiovascular Medicine, Tokushima University Hospital, Tokushima, Japan

13 Department of Cardiology, Kimitsu Chuo Hospital, Kisarazu, Japan

14 Department of Cardiology, Mashiko Hospital, Kawaguchi, Japan

15 Department of Cardiovascular Medicine, Kitasato University School of Medicine, Sagamihara, Japan 


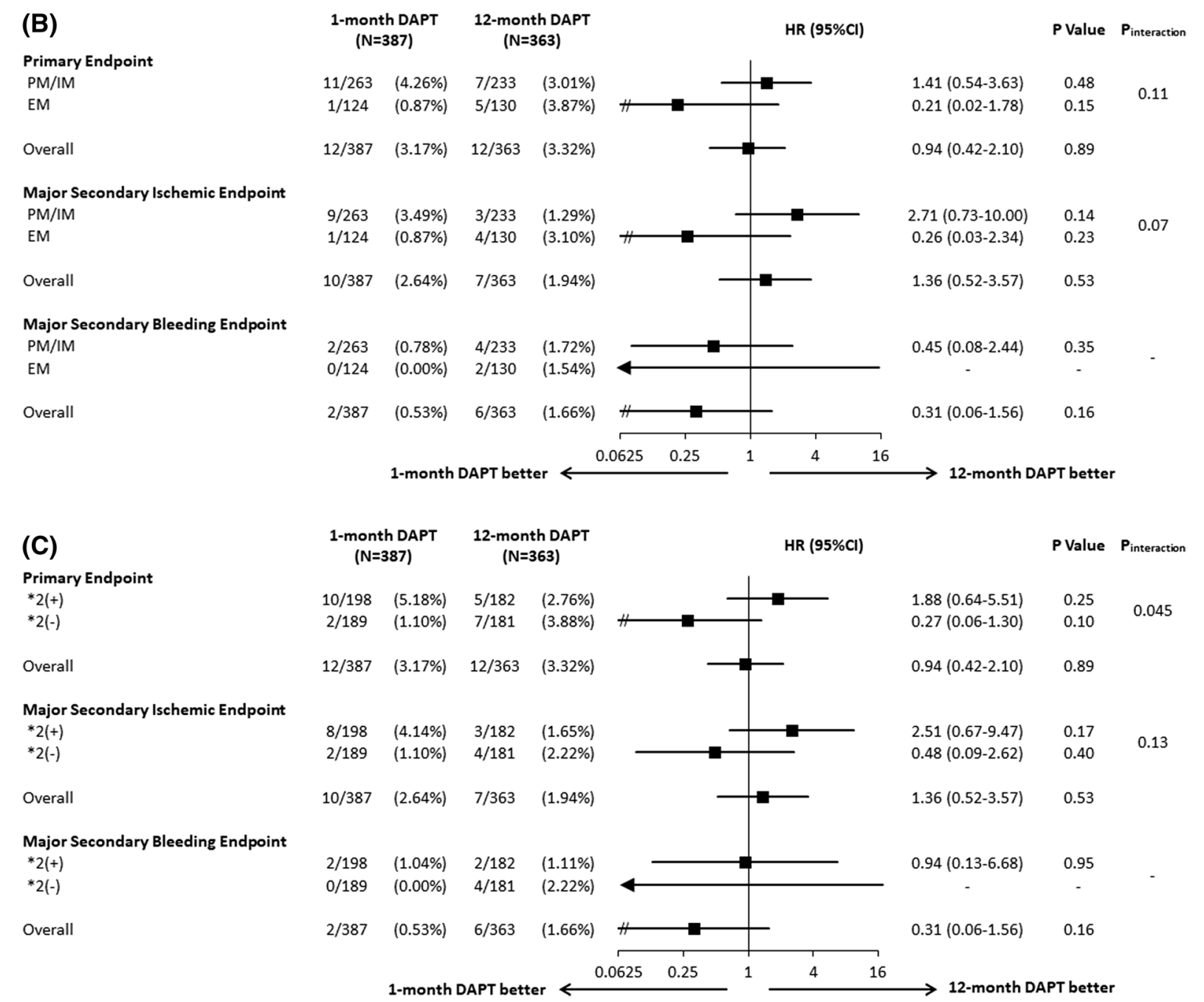

Fig. 4 The effect of 1-month DAPT relative to 12-month DAPT stratified by CYP2C19 genotypes. Effects of 1-month versus 12-month DAPT for the primary and major secondary endpoints stratified by a three CYP2C19 genotypes (PM, IM, and EM), b PM/IM and EM (with or without CYP2C19*2 or $* 3$ LOF allele), $\mathbf{c}$ with or without
CYP2C19*2 LOF allele, and d with or without CYP2C19*3 LOF allele. CI, confidential interval; DAPT, dual antiplatelet therapy; EM, extensive metabolizer; HR, hazard ratio; IM, intermediate metabolizer; LOF, loss-of-function; and PM, poor metabolizer

Publisher's Note Springer Nature remains neutral with regard to jurisdictional claims in published maps and institutional affiliations. 